Original article

\title{
Investigating the shear bond strength of five resin-based luting agents to zirconia ceramics
}

\author{
Xiuju Liu, Xue Jiang, Tong Xu, Qi Zhao, and Song Zhu \\ Department of Prosthodontics, School and Hospital of Stomatology, Jilin University, Jilin, P. R. China
}

(Received December 25, 2018; Accepted June 6, 2019)

\begin{abstract}
The present study investigated the bond strength and aging resistance performance of five resin-based luting agents to zirconia. A total of 100 large blocks $(10.0 \mathrm{~mm} \times 10.0 \mathrm{~mm} \times 2.5 \mathrm{~mm})$ and 100 small blocks $(4.0 \mathrm{~mm} \times 4.0 \mathrm{~mm} \times 2.5 \mathrm{~mm})$ of zirconia were airborne-particle abraded and randomly divided into five groups: (RelyX Ultimate [RUl]; Panavia F [PF]; Clearfil SA Luting [SAC]; Multilink Speed [MS]; and RelyX Unicem [RUn]). The small blocks were bonded to the large blocks using the resin-based luting agents. Shear bond strengths (SBS) and failure mode were determined before and after 5,000 thermocycles. After being stored in water for $24 \mathrm{~h}$, the SBS were MS $>\mathrm{PF}>\mathrm{RUl}>\mathrm{SAC}>\mathrm{RUn}(P<0.05)$. After 5,000 thermocycles, the SBS were MS $>\mathrm{SAC} \approx \mathrm{RUl}>\mathrm{PF} \approx \mathrm{RUn}$ $(P<0.05)$; the SBS of the PF, MS, and RUn groups were lower than that before 5,000 thermocycles $(P<0.01)$. Adhesive failure, cohesive failure, or mixed failure occurred in the specimens. In general, Clearfil SA Luting, a self-adhesive resin-based luting agent containing 10-methacryloxy decyl diphosphate, had good initial and durable SBS to zirconia and was a better adhesive.
\end{abstract}

Keywords; 10-MDP, resin-based luting agents, SBS, zirconia

\section{Introduction}

Because of its unique stress-induced phase-transformation effects, yttriastabilized tetragonal zirconia polycrystalline (Y-TZP) possesses high fracture toughness and strength, excellent esthetics, and good biocompatibility. In recent years, it has been widely applied in prosthodontics and implant dentistry, particularly for inlays, crowns, and implant substrates [1].

Y-TZP is a polycrystalline, high-strength oxide ceramic without a glassy phase. Because there is no silicon dioxide structure on the surface, it has a high chemical inertia. Bonding to zirconia, however, is problematic. To obtain high bond strength and maintain bonding durability, some type of mechanical and chemical pretreatment of the zirconia surface-such as alumina airborne-particle abrasion, selective acid-etching, or tribochemical silicon coating - and primer application is necessary [2,3]. Although there is no universally accepted method of improving clinical bond strength, alumina airborne-particle abrasion usually produces the micromechanical retention that is advocated for in clinical applications [4]. However, airborne particles impacting the surface of zirconia may induce small cracks or defects, which may lead to fracture during oral functions such as mastication or tooth grinding. Airborne-particle abrasion may also induce the transformation of zirconia from the tetragonal to monoclinic phase, thus affecting its long-term properties [5]. In view of this problem, the use of a resin adhesive that can fill the microcracks caused by airborne-particle abrasion has been advocated to increase the strength of zirconia [6].

The preferred adhesive for zirconia restorations is a resin-based luting agent. The bonding mechanism mainly includes a chemical and micromechanical bonding force between the roughened and pretreated zirconia surface and the resin-based luting agent. Chemical bonding has been shown to play a decisive role in the bond strength and durability of zirconia. Some resin-based luting agents for zirconia bonding contain functional

Correspondence to Dr. Song Zhu, School and Hospital of Stomatology, Jilin University, No.1500 Qinghua Road, Chaoyang District, Changchun, Jilin 130021, P. R. China

Fax:+86-431-88796038 E-mail: zhusong1965@163.com

Color figures can be viewed in the online issue at J-STAGE.

doi.org/10.2334/josnusd.18-0480

DN/JST.JSTAGE/josnusd/18-0480 monomers that have an affinity for and can react with zirconia to improve the bond between the resin-based luting agent and zirconia.

Because of the chemical interaction between the phosphate ester group of these molecules and the hydroxyl groups of the passive zirconia surface, resin-based luting agents containing phosphate-based functional monomers, particularly 10-methacryloxydecyl dihydrogen phosphate (10MDP), seem to be a suitable adhesive for zirconia bonding [7]. However, conventional resin-based luting agents without functional monomers combined with other adhesives also have been used for bonding to zirconia [8,9]. Conventional resin-based luting agents combined with primer containing 10-MDP or a general binder can also improve the bond strength to zirconia [10]. Therefore, the present bonding strategy of combining the application of airborne-particle abrasion and primer or a resin-based luting agent containing acidic functional monomers (e.g., 10-MDP) is a preferred method for zirconia bonding [11,12].

The initial bond strength between a resin-based luting agent and existing tooth structure is between $17 \mathrm{MPa}$ and $20 \mathrm{MPa}$, which meets clinical requirements. However, the bond strength between a resin-based luting agent and zirconia is poor and needs to be improved [13]. A long-term, stable bond between resin and zirconia ensures a marginal seal, prevents fracture, and improves the success rate of a prosthesis [14]. However, studies on the aging resistance of resin-based luting agents bonded to zirconia are lacking.

The purpose of this study was to compare the effects of five resin-based luting agents on shear bond strength to investigate their suitability for bonding to zirconia. The operation flow of this experiment is shown in Fig. 1.

\section{Materials and Methods}

\section{Preparation of specimens}

A total of 100 large zirconia blocks $(10.0 \mathrm{~mm} \times 10.0 \mathrm{~mm} \times 2.5 \mathrm{~mm})$ and 100 small zirconia blocks $(4.0 \mathrm{~mm} \times 4.0 \mathrm{~mm} \times 2.5 \mathrm{~mm})$ were prepared from zirconia (Cercon, DeguDent GmbH, Hanau-Wolfgang, Germany) in a cutting machine (SYJ-160, Shenyang Kejing Co Ltd, Shenyang, P. R. China). The specimens were sintered according to the manufacturer's instructions. The zirconia blocks were polished with 600-, 800-, and 1,000-grit silicon carbide abrasive papers for $10 \mathrm{~s}$ under running water. A selected surface of each block was airborne-particle abraded with $50-\mu \mathrm{m}$ aluminum oxide (Rocatec, 3M ESPE, St. Paul, MN, USA) perpendicularly to the zirconia surface ( $0.25 \mathrm{MPa}$ pressure, $10-\mathrm{mm}$ distance, $10 \mathrm{~s})$. They were then ultrasonically cleaned in acetone, ethanol, and deionized water in an ultrasonic bath (Eurosonic Energy, Euronda Inc., Vicenza, Italy) for 5 min and rinsed with distilled water for $30 \mathrm{~s}$. The specimens were randomly divided into 5 groups, each including 20 large blocks and 20 small blocks.

The specific grouping and the information on each adhesive (RelyX Ultimate [RU1], 3M, St. Paul, MN, USA; Panavia F [PF], Kuraray, Tokyo, Japan; Clearfil SA Luting [SAC], Kuraray, Tokyo, Japan; Multilink Speed [MS], Ivoclar Vivadent AG, Liechtenstein; and RelyX Unicem [RUn], 3M) is shown in Table 1. The prepared large blocks were placed in autopolymerizing resin with the bonding surface sufficiently exposed. The resin-based luting agents were placed on the small blocks following the respective manufacturer's instructions (Table 1), which were then bonded to the large blocks under a pressure of $10 \mathrm{~N}$. The excess cement was removed after light-polymerization for 3-5 s using a light-curing machine (SLC-VIIIA, Hangzhou Quartet Medical Devices Co Ltd, Hangzhou, P. R. China) with $900 \mathrm{~mW} / \mathrm{cm}^{2}$ light-power density. After the pressure and the excess cement had been removed, the specimens were light-polymerized from 2 opposite 


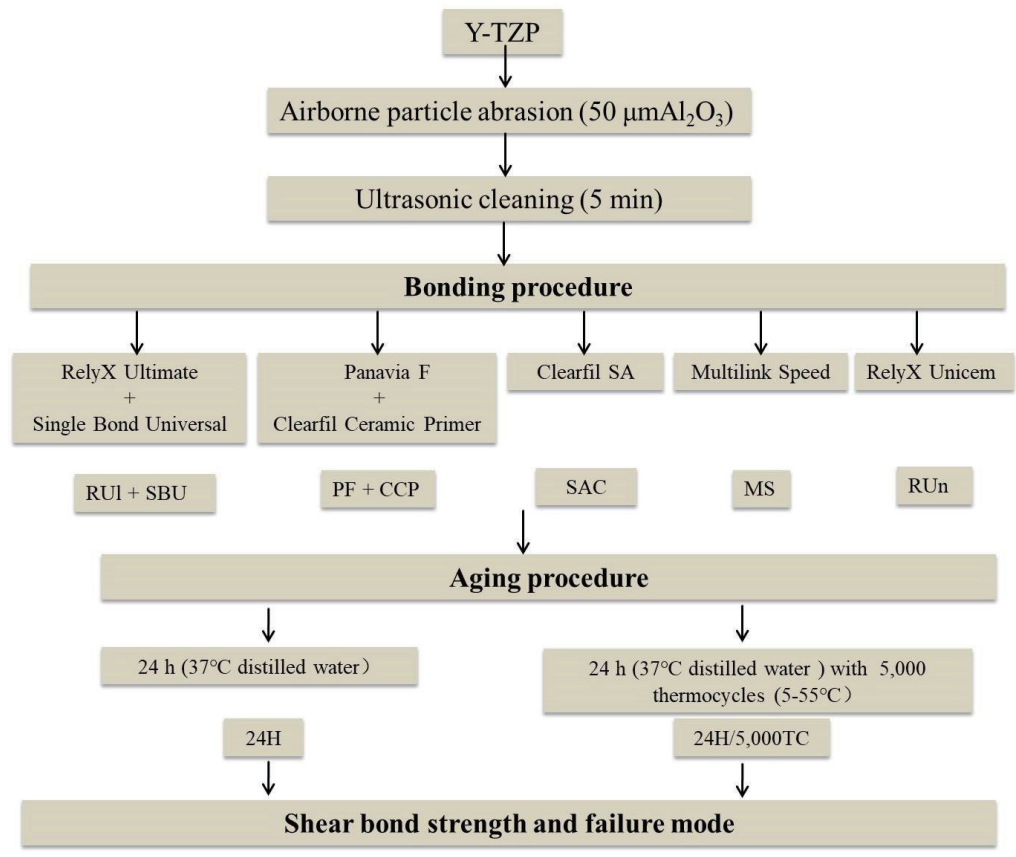

Fig. 1 Experimental design $(n=10)$

Table 1 Materials evaluated

\begin{tabular}{|c|c|c|c|}
\hline Material & Manufacturer & Main composition* & Application procedure \\
\hline $\begin{array}{l}\text { RelyX Ultimate } \\
\text { (RUl) }\end{array}$ & $\begin{array}{l}3 \mathrm{M}, \\
\text { St, Paul, MN, } \\
\text { USA }\end{array}$ & $\begin{array}{l}\text { Silane-treated glass powder, 2-propenoicacid, 2-methyl-1, 10[1-(hydroxymethyl)1,2- } \\
\text { ethanediyl] ester, reaction products with 2-hydroxy-1,3-propanediyl dimethacrylate and } \\
\text { phosphorus oxide, TEGDEMA, silane-treated silica, oxide-glass chemicals, sodium } \\
\text { persulfate, tert-butyl peroxy-3,5,5-trimethylhexanoate, copper, acetate monohydrate, } \\
\text { substituted dimethacrylate,1,12-dodecane dimethacrylate, 1-benzyl-5-phenyl-barbic-acid, } \\
\text { calcium salt, sodium p-toluenesulfonate, calcium hydroxide, 2-propenoic acid, 2-methyl- } \\
\text { [(3methoxypropyl)imino] di-2,1-ethanediyl ester, titanium dioxide }\end{array}$ & $\begin{array}{l}\text { 1. Apply Single Bond Universal Adhesive on the } \\
\text { surface of specimens for } 20 \mathrm{~s} \text {, then thoroughly } \\
\text { air-dried. } \\
\text { 2. Squeeze Paste A and B from the dispenser syringe } \\
\text { and mix sufficiently on the paper pad for } 20 \mathrm{~s} \text {. } \\
\text { 3. Apply the mixed RUl to small blocks. } \\
\text { 4. Light cure for } 20 \mathrm{~s} \text { from each side. }\end{array}$ \\
\hline $\begin{array}{l}\text { Single Bond } \\
\text { Universal }\end{array}$ & $3 \mathrm{M}$ & $\begin{array}{l}\text { HEMA, Bis-GMA, absolute ethyl alcohol, 2-methyl -2-acrylic -1,10- decyl } 2 \text { ester, } 2 \text { - methyl } \\
\text {-2- acrylic acid, 1,10- decanediol, a mixture of phosphorus pentoxide, silane silicide mol- } \\
\text { ecules, water }\end{array}$ & \\
\hline $\begin{array}{l}\text { Panavia F2.0 } \\
(\mathrm{PF})\end{array}$ & $\begin{array}{l}\text { Kuraray, } \\
\text { Tokyo, Japan }\end{array}$ & $\begin{array}{l}\text { hydrophobicaromatic DMA, hydrophobicaliphatic DMA, hydrophilic DMA, 10-MDP, siben- } \\
\text { zoyl peroxide containing composite resin, silanated silica, silanated barium glass-containing } \\
\text { composite resin, } N, N \text {-diethanol- } p \text {-toluidine, surface-treated (functionalized) sodium fluoride, } \\
\text { photoinitiator }\end{array}$ & $\begin{array}{l}\text { 1. Apply primer on the surface of specimens for } 5 \mathrm{~s} \text {, } \\
\text { then thoroughly air-dry. } \\
\text { 2. Dispense equal amounts of Paste A and B and mix } \\
\text { sufficiently on the paper pad for } 20 \mathrm{~s} \text {. }\end{array}$ \\
\hline $\begin{array}{l}\text { Clearfil Ceramic } \\
\text { Primer }\end{array}$ & Kuraray & 10-MDP, 3-(trimethoxysilyl) propyl methacrylate, ethanol & $\begin{array}{l}\text { 3. Apply the mixed Panavia } F \text { to small blocks. } \\
\text { 4. Light cure for } 20 \mathrm{~s} \text { from each side. }\end{array}$ \\
\hline $\begin{array}{l}\text { Multilink Speed } \\
\text { (MS) }\end{array}$ & $\begin{array}{l}\text { Ivoclar Vivadent, } \\
\text { Schaan, } \\
\text { Liechtenstein }\end{array}$ & $\begin{array}{l}\text { 10-MDP, 5-NMSA, } \mathrm{SiO}_{2}, \mathrm{HEMA} \text {, barium-treated glass powder, sodium fluoride, benzoyl } \\
\text { peroxide }\end{array}$ & $\begin{array}{l}\text { 1. Squeeze Paste A and B from the dispenser syringe } \\
\text { and mix sufficiently on the paper pad for } 20 \mathrm{~s} \text {. } \\
\text { 2. Apply the mixed resin cements to small blocks. }\end{array}$ \\
\hline $\begin{array}{l}\text { Clearfil SA resin } \\
\text { cement } \\
(\mathrm{SAC})\end{array}$ & Kuraray & $\begin{array}{l}\text { Bis-GMA, sodium fluoride, TEGDMA, 10-MDP, hydrophobic aromatic dimethacrylate, } \\
\text { hydrophobic aliphatic dimethacrylate, silanated barium glass filler, silanated colloidal silica, } \\
d l \text {-camphorquinone, initiators, accelerators, catalysts, pigments }\end{array}$ & $\mathrm{t}$ cure for $20 \mathrm{~s}$ from each side. \\
\hline $\begin{array}{l}\text { RelyX Unicem } \\
\text { (RUn) }\end{array}$ & $3 \mathrm{M}$ & $\begin{array}{l}\text { methacrylated phosphoric esters, dimethacrylates, acetate, initiators, stabilizers, glass fillers, } \\
\text { silica, calcium hydroxide }\end{array}$ & $\begin{array}{l}\text { 1. The capsule was activated by an activator for } 2 \mathrm{~s} \\
\text { to } 4 \mathrm{~s} \text { and mixed with a mixing machine for } 10 \mathrm{~s} \text {. } \\
\text { 2. Apply the mixed RUn to small blocks. } \\
\text { 3. Light cure for } 20 \mathrm{~s} \text { from each side. }\end{array}$ \\
\hline
\end{tabular}

*According to the manufacturers' fact sheets and instructions. 5-NMSA, $N$-methacryloyl-5-aminosalicylic acid; 10-MDP, 10-methacryloxydecyl dihydrogen phosphate; Bis-GMA, bisphenol A-glycidyl methacrylate; DMA, dimethacrylate; HEMA, hydroxyethyl methacrylate; $\mathrm{SiO}_{2}$, silicon dioxide; TEGDMA, tri ethylene glycol dimethacrylate

margins of the block for $20 \mathrm{~s}$ each.

After 30 min at room temperature, the specimens were stored in distilled water at $37^{\circ} \mathrm{C}$ for $24 \mathrm{~h}$; one half was then tested $(24 \mathrm{H})$ and the other half was thermocycled (Proto-Tech, Micoforce, Portland, OR, USA) for 5,000 cycles with alternating temperatures of $5^{\circ} \mathrm{C}$ and $55^{\circ} \mathrm{C}(24 \mathrm{H} / 5,000 \mathrm{TC})$.

\section{Scanning electron microscopy observation}

The surface of the zirconia was well coated after drying for 5 min under a digital infrared baking lamp. The surface structure of zirconia before and after airborne-particle abrasion was observed with a scanning electron microscope (SEM) (S-4800, Hitachi Ltd, Tokyo, Japan).

\section{Shear bond strength test}

The shear bond strength (SBS) was measured with a universal testing machine (AG-XPLUS 10KN, Shimadzu Co Ltd., Kyoto, Japan) with a
$1-\mathrm{kN}$ load cell at a crosshead speed of $1 \mathrm{~mm} / \mathrm{min}$ until failure occurred. The bonded specimens were fixed in a custom fixture and the plane of the loading head was parallel to the bonding surface (Fig. 2). The SBS was calculated using the following formula: $\mathrm{P}=\mathrm{F} / \mathrm{S}$ [15], where $\mathrm{P}$ was the shear bond strength $(\mathrm{MPa})$; F was the maximum shear force $(\mathrm{N})$; and $\mathrm{S}$ was the bonding area $\left(\mathrm{mm}^{2}\right)$.

\section{Failure mode observation}

The failure mode was determined with a stereoscopic microscope (SZX16, OLYMPUS Co Ltd., Tokyo, Japan) under $\times 20$ magnification. Failure was classified by the following method:

A. Interface failure: A fracture occurred at either the interface between the resin-based luting agents and zirconia, one zirconia surface without resin-based luting agents, or all of the resin-based luting agents on the other zirconia surface; 


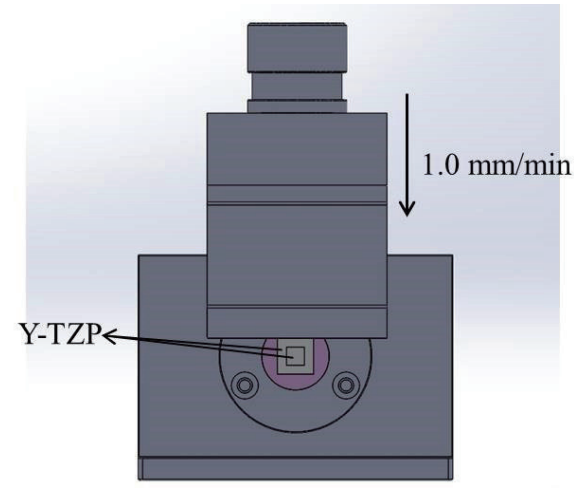

Fig. 2 Schematic diagram of shear strength test
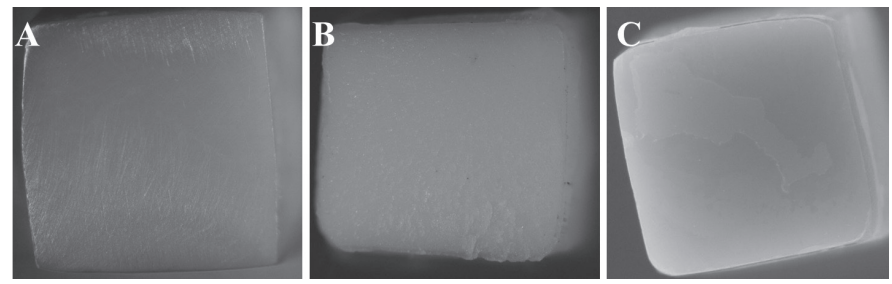

Fig. 4 Failure modes of specimens. A, interfacial failure; B, adhesive cohesive failure; C, mixed failure

Table 3 Classification of fracture modes

\begin{tabular}{llllll}
\hline Groups & I & II & III & IV \\
\hline RUl & $24 \mathrm{H}$ & 0 & 0 & 5 & 5 \\
& $24 \mathrm{H} / 5,000 \mathrm{TC}$ & 0 & 0 & 4 & 6 \\
PF & $24 \mathrm{H}$ & 0 & 0 & 7 & 3 \\
\multirow{3}{*}{$\mathrm{SAC}$} & $24 \mathrm{H} / 5,000 \mathrm{TC}$ & 0 & 0 & 6 & 4 \\
& $24 \mathrm{H}$ & 0 & 0 & 8 & 2 \\
\multirow{3}{*}{$\mathrm{MS}$} & $24 \mathrm{H} / 5,000 \mathrm{TC}$ & 0 & 0 & 5 & 5 \\
\multirow{3}{*}{ RUn } & $24 \mathrm{H}$ & 3 & 0 & 5 & 2 \\
& $24 \mathrm{H} / 5,000 \mathrm{TC}$ & 0 & 0 & 4 & 6 \\
& $24 \mathrm{H}$ & 0 & 0 & 6 & 4 \\
\hline
\end{tabular}

Group I adhesive cohesive failure; Group II zirconia cohesive failure; Group III interfacial failure; Group IV-mixed failure

B. Cohesive failure: There was an internal fracture of zirconia or a resinbased luting agent fracture where the surface of two zirconia specimens was covered by resin-based luting agents;

C. Mixed failure: At the same time, there was interface damage and cohesive failure and the surface of two zirconia specimens was partially covered by resin-based luting agents.

\section{Statistical analysis}

Data were described as mean \pm standard deviation. After the data were accorded with normal probability distribution and homogeneity of variance using the Kolmogolov-Smirnov test and Levene's test, a two-way analysis of variance was conducted to evaluate the effect of diverse resin-based luting agents and different experimental conditions on SBS. Tukey honest significant difference was applied to compare the experimental groups $(\alpha=$ 0.05). The statistical analyses were performed with Statistical Package for the Social Sciences (SPSS) statistical software (SPSS version 22.0, SPSS Inc, Chicago, IL, USA).

\section{Results}

SEM (Fig. 3) showed that the surface of zirconia before airborne-particle abrasion had a certain roughness, but porosity was not detected. After airborne-particle abrasion, the surface of zirconia ceramics presented ridges or grains, and the roughness appeared to increase significantly.

The results of Kolmogolov-Smirnov and Levene's tests showed that the data were accorded with normal probability distribution and homogeneity of variance $(P>0.05)$. The SBS values of all test groups are presented in
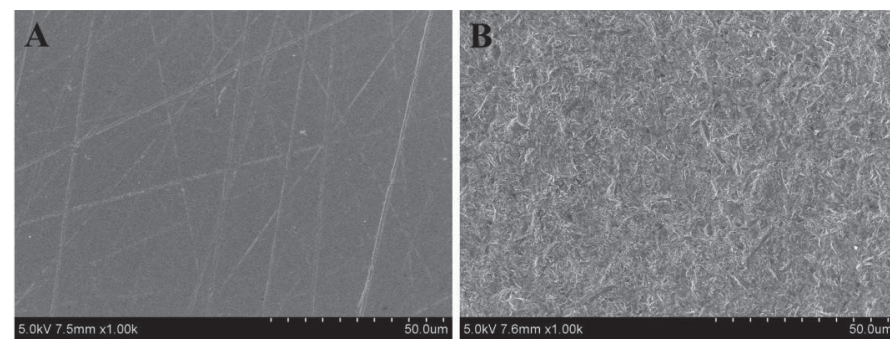

Fig. 3 Scanning electron microscopy of zirconia $(\times 1,000)$. A, before airborne-particle abrasion; B, after airborne-particle abrasion

Table 2 SBS values of specimens $(\mathrm{X} \pm \mathrm{S}, n=10)$

\begin{tabular}{lcc}
\hline \multirow{2}{*}{ Groups } & \multicolumn{2}{c}{ SBS $(\mathrm{MPa})$} \\
\cline { 2 - 3 } & $24 \mathrm{H}$ & $24 \mathrm{H} / 5,000 \mathrm{TC}$ \\
\hline RUl & $22.83 \pm 1.54^{\mathrm{Aa}}$ & $20.11 \pm 1.73^{\mathrm{Aa}}$ \\
PF & $24.35 \pm 1.45^{\mathrm{Ba}}$ & $13.84 \pm 1.02^{\mathrm{Bb}}$ \\
SAC & $20.59 \pm 1.00^{\mathrm{Ca}}$ & $20.13 \pm 0.88^{\mathrm{Aa}}$ \\
MS & $33.70 \pm 0.92^{\mathrm{Da}}$ & $21.29 \pm 0.82^{\mathrm{Cb}}$ \\
RUn & $17.19 \pm 1.12^{\mathrm{Ea}}$ & $13.74 \pm 1.09^{\mathrm{Bb}}$ \\
\hline
\end{tabular}

The superscripted letters indicate significant differences within the same column $(P<0.05)$; the different lowercase letters indicate significant differences within the same line $(P<0.05)$.

Table 2. After water storage for $24 \mathrm{~h}$, the SBS values of the 5 cements ranging from large to small were: $\mathrm{MS}>\mathrm{PF}>\mathrm{RUl}>\mathrm{SAC}>\mathrm{RUn}(P<0.05)$. After 5,000 cycles, the SBS values were: $\mathrm{MS}>\mathrm{SAC} \approx \mathrm{RUl}>\mathrm{PF} \approx \mathrm{RUn}$ $(P<0.05)$. Compared with $24 \mathrm{H}$, the SBS values of the PF, MS, and RUn groups were lower after 5,000 cycles $(P<0.01)$, but the SBS values of RUl and SAC groups did not change significantly $(P>0.05)$.

The fracture surface observation by stereomicroscope (Fig. 4, Table 3) showed that cohesive failure of the adhesive occurred only in the MS group after $24 \mathrm{~h}$ and that interfacial fracture and mixed fracture occurred in specimens of the other test groups.

\section{Discussion}

Owing to the high chemical inertia of Y-TZP, surface modification is required to obtain micromechanical retention and/or chemical bonding. Alumina airborne-particle abrasion can promote micromechanical bonding between zirconia ceramics and resin-based luting agents by removing the smear layer, roughening the zirconia surface, increasing the surface area for bonding, and improving wettability [16]. The SEM images show that the surface of zirconia has a certain roughness before airborne-particle abrasion but that porosity is not visible. After airborne-particle abrasion, the surface porosity increases. In addition, alumina airborne-particle abrasion can significantly change the surface morphology and structure of zirconia, resulting in tetragonal $(\mathrm{t})$ to monoclinic $(\mathrm{m})$ phase transition; this is conducive to the formation of micromechanical or chemical bonding between zirconia and the tooth [17]. Airborne-particle abrasion is the most convenient and effective way of improving the bond strength to zirconia ceramics $[18,19]$.

The adhesives used for zirconia restorations in clinical settings are mainly resin-based luting agents, and can be divided into three systems according to their processing method: total-etch, self-etch, or self-adhesive. The functional monomers of resin-based luting agents can improve chemical bonding between the cement and zirconia by forming chemical bonds with metal oxides. In addition, van der Waals forces or hydrogen bonds form secondary internal forces at the resin-zirconia interface. At the same time, the surface wettability and bond strength of zirconia are improved. At present, 10-MDP is the most widely used functional monomer [7,20]. In this study, the five resin-based luting agents were selected according to their different adhesive systems and whether they contained 10-MDP: total-etch, resin-based luting agent RUl and self-adhesive, resin-based luting agent RUn without 10-MDP; self-etch, resin-based luting agent PF and self-adhesive, resin-based luting agents SAC, and MS with 10-MDP.

10-MDP is an acidic monomer with high affinity for metal oxides such as zirconia. It can provide dual-function adhesion between metal/metal oxides and the bisphenol A-glycidyl methacrylate (Bis-GMA) resin matrix [20], dissolve the microparticle layer, and penetrate the ceramic through 
diffusion, resulting in a resin-infiltrated layer on the surface of the zirconia and improving the bond strength. It can also be chemically conditioned to create an "active" zirconia surface, facilitating the formation of chemical bonds between its phosphate groups and the oxides on the zirconia surface [21]. Furthermore, 10-MDP can react directly with the hydroxyl groups on the surface of zirconia and produce van der Waals force or hydrogen bonds to form stable hydrolysis-resistant chemical bonds, which improves the durability of the bond strength $[22,23]$. The hydrophilic phosphate group in the 10-MDP molecule can etch and demineralize natural teeth and combine with calcium ions or amino acids to form insoluble calcium salts which bond well with natural teeth, especially dentin. Many studies have shown that adhesives containing the 10-MDP monomer have higher bond strengths than traditional adhesives [24]. In this experiment, the SBS values of MS and PF, which contain 10-MDP, was significantly higher than those of RUl and RUn after water storage for $24 \mathrm{~h}$.

In fact, after water storage for $24 \mathrm{~h}$, the SBS values of MS, PF, and SAC cements containing 10-MDP were significantly different. This may be because the ability of the adhesive to bond with zirconia is not only associated with the chemical composition of the adhesive but is also affected by its wettability and viscosity. Low-viscosity resin-based luting agents can easily flow into the pores of the sandblasted zirconia surface, increasing the bonding surface. However, when high-viscosity resin-based luting agents are used, the acid-etching effect is poor and the fluidity is significantly reduced after illumination, decreasing the micromechanical locking effect between the resin-based luting agent and the bonding surface of zirconia [25]. Further study of different 10-MDP containing resin cements should be conducted.

Due to the presence of 10-MDP monomers, SAC should have higher bond strengths than RUl, but the result was contradictory. It might be caused by Single Bond Universal (3M) in which 10-MDP was used to further improve the bond strength with zirconia before RUl application. However, RUn did not contain the functional bonding monomer 10-MDP, and the chemical bond strength with zirconia was weak. Therefore, its bond strength was lower. These results were in agreement with other studies [Feng XL et al., Key Engineering Materials, 602-603, 602-605, 2014].

Evaluating the aging resistance of the adhesive system in the complex oral environment is of great clinical significance. In this study, thermocycling experiments were performed to evaluate the bonding durability of the five resin-based luting agents. The results showed that the SBS value of SAC did not decrease after thermocycling $(P>0.05)$, which may be attributed to the higher content of 10-MDP. However, the SBS values of MS and PF containing 10-MDP decreased significantly after 5,000 cycles $(P<0.01)$. This may be related to the film thickness, water absorption, and solubility of resin-based luting agents [26]. If the film is too thick, the coefficient of thermal expansion between the resin-based luting agent and zirconia ceramics does not match, and a gap might occur after thermocycles, resulting in a decrease in bond strength. The self-adhesive resin-based luting agent contains an acidic adhesive monomer, and its water absorption value and dissolution value are higher than the conventional resin-based luting agent. When the resin-based luting agent absorbs water, it decreases the strength, expands the volume, and affects the durability of the bond [27]. Although MS had a significant decrease in SBS after 5,000 thermocycles, it was still higher than the other four resin-based luting agents. The bond strength after more than 5,000 thermocycles should be tested to clarify the bonding effect of MS. The initial bond strength is higher when the resin-based luting agent containing 10-MDP is used, but the bond strength decreases after artificial aging [28,29]. Therefore, the ability of adhesive systems containing 10-MDP to resist the loss of adhesive strength over time is still unclear [30,31].

This study found that the SBS values of RU1 did not decrease significantly after 5,000 cycles, which may be due to the use of Single Bond Universal before bonding. Single Bond Universal contains both 10-MDP and a methacrylic acid-modified polyolefin copolymer, which can effectively resist hydrolysis. This explains why the long-term bonding effect of the RUl group is higher than that of PF and RUn [32]. Self-adhesive, resin-based luting agents contain a phosphate monomer or a carboxylic acid-ester monomer, which is more hydrophilic than common dental resin monomers such as Bis-GMA $[33,34]$. During thermal cycling, water with a small molecular size and high molar concentration is more likely to lead to the dissolution and release of unpolymerized monomers, which softens the polymer and weakens its cross-linking structure, leading to filler overflow and eventually to the degradation of the resin-based luting agent [32]. Therefore, the better aging resistance of RUl and SAC may be attributed to the presence of the Bis-GMA monomer in RUl and SAC.

In addition, the difference in physical properties such as the thermal expansion coefficient, water absorption, and solubility of the resin-based luting agent will also affect the bonding interface [35]. During the thermal cycling process, the intermolecular force and the chemical bonding force between the bonding interface consisting of the ceramics and resin will be easily destroyed by water. Temperature changes may enlarge the mismatch of the thermal expansion coefficient of the bonding materials, resulting in mechanical stress at the bonding interface and bond strength degradation [36]. These factors can further aggravate the aging of the bonding interface, reducing the bond strength [37].

Analysis of the failure mode can help to explain bond strength results. In the present investigation, low bond-strength values were usually associated with cohesive failures [38]. After $24 \mathrm{~h}$ of water bath, only the MS group produced an adhesive failure, which coincided with the relatively high bond-strength values obtained. The other four groups produced cohesive failures or mixed failures, which further explains why the bond strength of the MS adhesive was the highest. After aging with 5,000 thermocycles, the failure patterns of all specimens were either cohesive or mixed.

In this experiment, SAC had good initial strength and it had more durable shear bond strength, which was greater than the $17 \mathrm{MPa}$ required for the teeth when in function [39]. Fahad et al. [40] compared the shear bond strength of three primer/resin cement systems to zirconia ceramic. The results revealed that the use of Clearfil SA/Clearfil Ceramic Primer system (Kuraray) based on 10-MDP could increase the bond strength of Y-TZP ceramics to core materials. Koizumi et al. [41] found that Clearfil SA Luting showed statistically higher bond strength than the other materials after 20,000 thermal cycles $(P<0.05)$. Therefore, Clearfil SA Luting is highly recommended for bonding zirconia ceramic restorations as a new double-curing, self-etching, and one-step resin adhesive.

\section{Acknowledgments}

This research was financially supported by the National Natural Science Foundation of China (NSFC, Grant No. 81671033). The authors thank Jilin Provincial Key Laboratory of Tooth Development and Bone and those who contributed to this article.

\section{Conflict of interest}

All authors have declared that they have no conflict of interest.

\section{References}

1. Solá-Ruíz MF, Agustin-Panadero R, Fons-Font A, Labaig-Rueda C (2015) A prospective evaluation of zirconia anterior partial fixed dental prostheses: clinical results after seven years. J Prosthet Dent 113, 578-584

2. Inokoshi M, Kameyama A, De Munck J, Minakuchi S, Van Meerbeek B (2013) Durable bonding to mechanically and/or chemically pre-treated dental zirconia. J Dent 41, 170-179.

3. Chen C, Xie H, Song X, Burrow MF, Chen G, Zhang F (2014) Evaluation of a commercial primer for bonding of zirconia to two different resin composite cements. J Adhes Dent 16, 169-176.

4. Thompson JY, Stoner BR, Piascik JR, Smith R (2011) Adhesion/cementation to zirconia and other non-silicate ceramics: Where are we now? Dent Mater 27, 71-82.

5. Guess PC, Zhang Y, Kim JW, Rekow ED, Thompson VP (2010) Damage and reliability of Y-TZP after cementation surface treatment. J Dent Res 89, 592-596.

6. Kim ST, Cho HJ, Lee YK, Choi SH, Moon HS (2010) Bond strength of Y-TZP-zirconia ceramics subjected to various surface roughening methods and layering porcelain. Surf Interface Anal 42, 576-580.

7. Papia E, Larsson C, Du TM, Vult von Steyern P (2014) Bonding between oxide ceramics and adhesive cement systems: a systematic review. J Biomed Mater Res B Appl Biomater $102,395-413$

8. de Sá Barbosa WF, Aguiar TR, Francescantonio MD, Cavalcanti AN, de Oliveira MT, Giannini M (2013) Effect of water storage on bond strength of self-adhesive resin cements to zirconium oxide ceramic. J Adhesive Dent 15, 145-150.

9. Aguiar TR, André CB, Corrersobrinho L, Arrais CA, Ambrosano GM, Giannini M (2014) Effect of storage times and mechanical load cycling on dentin bond strength of conventional and self-adhesive resin luting cements. J Prosthet Dent 111, 404-410.

10. Zhao L, Jian YT, Wang XD, Zhao K (2016) Bond strength of primer/cement systems to zirconia subjected to artificial aging. J Prosthet Dent 116, 790-796.

11. Mair L, Padipatvuthikul P (2010) Variables related to materials and preparing for bond strength testing irrespective of the test protocol. Dent Mater 26, e17-23.

12. Sciasci P, Abi-Rached FO, Adabo GL, Baldissara P, Fonseca RG (2015) Effect of surface treatments on the shear bond strength of luting cements to Y-TZP ceramic. J Prosthet Dent $113,212-219$

13. Barkmeier WW, Shaffer SE, Gwinnett AJ (1986) Effects of 15 vs 60 second enamel acid conditioning on adhesion and morphology. Oper Dent 11, 111-116. 
14. Yang L, Chen B, Xie H, Chen Y, Chen Y, Chen C (2018) Durability of Resin Bonding to Zirconia Using Products Containing 10-Methacryloyloxydecyl Dihydrogen Phosphate. J Adhes Dent 20, 279-287.

15. Piascik JR, Swift EJ, Braswell K, Stoner BR (2012) Surface fluorination of zirconia: adhesive bond strength comparison to commercial primers. Dent Mater 28, 604-608.

16. Subaşı MG, İnan Ö (2014) Influence of surface treatments and resin cement selection on bonding to zirconia. Lasers Med Sci 29, 19-27.

17. Yang B, Barloi A, Kern M (2010) Influence of air-abrasion on zirconia ceramic bonding using an adhesive composite resin. Dent Mater 26, 44-50.

18. Blatz MB, Phark JH, Ozer F, Mante FK, Saleh N, Bergler M et al. (2010) In vitro comparative bond strength of contemporary self-adhesive resin cements to zirconium oxide ceramic with and without air-particle abrasion. Clin Oral Investig 14, 187-192.

19. Shin YJ, Shin Y, Yi YA, Kim J, Lee IB, Cho BH et al. (2014) Evaluation of the shear bond strength of resin cement to Y-TZP ceramic after different surface treatments. Scanning 36, 479-486.

20. Thammajaruk P, Inokoshi M, Chong S, Guazzato M (2018) Bonding of composite cements to zirconia: a systematic review and meta-analysis of in vitro studies. J Mech Behay Biomed Mater 80, 258-268.

21. Fazi G, Vichi A, Ferrari M (2012) Influence of surface pretreatment on the short-term bond strength of resin composite to a zirconia-based material. Am J Dent 25, 73-78.

22. Inokoshi M, Poitevin A, De Munck J, Minakuchi S, Van Meerbeek B (2014) Bonding effectiveness to different chemically pre-treated dental zirconia. Clin Oral Investig 18, 1803-1812

23. Xie H, Tay FR, Zhang F, Lu Y, Shen S, Chen C (2015) Coupling of 10-methacryloyloxydecyldihydrogen phosphate to tetragonal zirconia: effect of $\mathrm{pH}$ reaction conditions on coordinate bonding. Dent Mater 31, e218-225.

24. Lee JJ, Choi JY, Seo JM (2017) Influence of nano-structured alumina coating on shear bond strength between Y-TZP ceramic and various dual-cured resin cements. J Adv Prosthodont 9, 130-137.

25. Moon JE, Kim SH, Lee JB, Ha SR, Choi YS (2011) The effect of preparation order on the crystal structure of yttria-stabilized tetragonal zirconia polycrystal and the shear bond strength of dental resin cements. Dent Mater 27, 651-663.

26. Lee JJ, Kang CK, Oh JW, Seo JM, Park JM (2015) Evaluation of shear bond strength between dual cure resin cement and zirconia ceramic after thermocycling treatment. J Adv Prosthodont 7, 1-7.

27. Ikemura K, Endo T (2010) A review of our development of dental adhesives - Effects of radical polymerization initiators and adhesive monomers on adhesion. Dent Mater J 29, $109-121$.
28. de Souza G, Hennig D, Aggarwal A, Tam LE (2014) The use of MDP-based materials for bonding to zirconia. J Prosthet Dent 112, 895-902

29. Tzanakakis EG, Tzoutzas IG, Koidis PT (2016) Is there a potential for durable adhesion to zirconia restorations? A systematic review. J Prosthet Dent 115, 9-19.

30. da Silva EM, Miragaya L, Sabrosa CE, Maia LC (2014) Stability of the bond between two resin cements and an yttria-stabilized zirconia ceramic after six months of aging in water. J Prosthet Dent 112, 568-575

31. Qeblawi DM, Campillo-Funollet M, Muñoz CA (2015) In vitro shear bond strength of two self-adhesive resin cements to zirconia. J Prosthet Dent 113, 122-127.

32. Durner J, Spahl W, Zaspel J, Schweikl H, Hickel R, Reichl FX (2010) Eluted substances from unpolymerized and polymerized dental restorative materials and their Nernst partition coefficient. Dent Mater 26, 91-99.

33. Kern M, Wegner SM (1998) Bonding to zirconia ceramic: adhesion methods and their durability. Dent Mater 14, 64-71.

34. Van Landuyt KL, Snauwaert J, De Munck J, Peumans M, Yoshida Y, Poitevin A et al. (2007) Systematic review of the chemical composition of contemporary dental adhesives. Biomaterials 28, 3757-3785

35. Marghalani HY (2012) Sorption and solubility characteristics of self-adhesive resin cements. Dent Mater 28, e187-198.

36. Gale MS, Darvell BW (1999) Thermal cycling procedures for laboratory testing of dental restorations. J Dent 27, 89-99.

37. Inokoshi M, De Munck J, Minakuchi S, Van Meerbeek B (2014) Meta-analysis of bonding effectiveness to zirconia ceramics. J Dent Res 93, 329-334.

38. de Castro HL, Corazza PH, Paes-Júnior T de A, Della Bona A (2012) Influence of Y-TZP ceramic treatment and different resin cements on bond strength to dentin. Dent Mater 28, 1191-1197.

39. Gomes AL, Castillo-Oyagüe R, Lynch CD, Montero J, Albaladejo A (2013) Influence of sandblasting granulometry and resin cement composition on microtensile bond strength to zirconia ceramic for dental prosthetic frameworks. J Dent 41, 31-41.

40. Al-Harbi FA, Ayad NM, Khan ZA, Mahrous AA, Morgano SM (2016) In vitro shear bond strength of Y-TZP ceramics to different core materials with the use of three primer/resin cement systems. J Prosthet Dent 115, 84-89.

41. Koizumi H, Nakayama D, Komine F, Blatz MB, Matsumura H (2012) Bonding of resinbased luting cements to zirconia with and without the use of ceramic priming agents. J Adhes Dent 14, 385-392. 